\author{
Jan Oosterhof \\ Theo M. De Boo \\ Rob A.B. Oostendorp \\ Oliver H.G. Wilder-Smith \\ Ben J.P. Crul
}

\section{Outcome of transcutaneous electrical nerve stimulation in chronic pain: short-term results of a double-blind, randomised, placebo-controlled trial}

Received: 11 April 2006

Accepted in revised form: 14 June 2006

Published online: 11 August 2006

J. Oosterhof $(\square)$

Research Centre for Allied Health Care, 645 Department of Physiotherapy CSS, Radboud University Nijmegen Medical Centre,

Geert Grooteplein 10, P.O. Box 9101, 6500 HB Nijmegen, The Netherlands e-mail: j.oosterhof@ fysiocss.umcn.nl Tel.: +31-24-3613811

Fax: +31-24-3619404

J. Oosterhof • O.H.G. Wilder-Smith

B.J.P. Crul

Pain Centre,

Department of Anesthesiology,

Radboud University Nijmegen

Medical Centre,

Nijmegen, The Netherlands

T.M. De Boo

Department of Epidemiology \&

Biostatistics,

Radboud University Nijmegen

Medical Centre,

Nijmegen, The Netherlands

R.A.B. Oostendorp

Research Centre for Allied Health Care, Department of Quality of Care Research, Radboud University Nijmegen Medical Centre,

Geert Grooteplein 10,

6500 HB Nijmegen, The Netherlands

R.A.B. Oostendorp

National Institute for Allied Health Care, Amersfoort, The Netherlands

\begin{abstract}
The aim of this study was to test the efficacy of shortterm transcutaneous electrical nerve stimulation (TENS) treatment in chronic pain with respect to pain intensity and patients' satisfaction with treatment results. We therefore performed a randomised controlled trial comparing TENS and sham TENS. Patients, researchers and therapists were blinded for treatment allocation. One hundred and sixty-three patients with chronic pain referred to the Pain Centre entered the study. Conventional TENS and sham TENS were applied in the segments of pain, for a period of ten days. Outcome measures were pain intensity (visual analogue scale) and patients' satisfaction with treatment result (yes or no). The proportions of patients satisfied with treatment result differed significantly for TENS compared to sham TENS (58 and $42.7 \%$ respectively, $x^{2}=3.8, p=0.05$ ). However, no differences in pain intensity were found for patients treated with TENS or sham TENS. Only for patients satisfied with treatment results pain intensity gradually decrease equally both for TENS and sham TENS with repeated treatment application.
\end{abstract}

Key words Transcutaneous electric nerve stimulation $\cdot$ Chronic pain - Patient satisfaction - Placebo effect $\cdot$ Duble-blind randomized controlled trial 


\section{Introduction}

Transcutaneous electrical nerve stimulation (TENS) is an easy to use, non-invasive intervention that alleviates pain. Although TENS treatment has been used since the early 1970 s, results of treatment of patients with chronic pain are still inconclusive $[1,2]$. These unclear results may be due to the fact that TENS treatment was applied intermittently for only short periods with stimulation time of less than $10 \mathrm{~h} /$ week [2], in different poorly defined modalities [1]. This treatment is of shorter duration and stimulation time than recommended treatments [3]. Furthermore, the use of different pain outcome measures gave contrary results, e.g., in patients with chronic low back pain, Moore and Shurman [4] found a significant difference in pain relief for TENS treatment as measured by a pain relief scale, but documented no pain reduction using a visual analogue scale (VAS), both compared with placebo, after two days of treatment. So patients did experience relief after this short treatment period, although this could not be retrieved by diminished VAS scores.

In exploring the effect of TENS treatment in chronic pain, measuring pain intensity by VAS [1] and calculating group means is very common to evaluate differences between TENS and sham TENS, assuming continuous and normally distributed data. However, situations might arise in which a particular treatment application produces a substantial benefit in a moderate proportion of patients, but no change in others. In this case, it is important to know that in animal research high-frequency TENS is effective in reducing hyperalgesia in the inflammatory pain model in rats [5], but not in a rat model of peripheral nerve ligation [6]. Also, for placebo response it is assumed that patients either do respond or do not respond [7]. For bimodal data, when there is little or no difference in group mean values, it is still possible that there might be a statistically significant difference in proportions of patients achieving a clinically important benefit, as proposed by Farrar et al. [8]. A problem may arise when trying to define "a clinically important improvement" for the result of TENS treatment when TENS is applied for short periods. A 50\% change in pain intensity has been used to evaluate TENS treatment effect in patients with osteoarthritis; Smith et al. [9] found $50 \%$ or more relief in pain intensity in $67 \%$ and $27 \%$ of the patients treated with TENS and sham TENS respectively; treatment was applied 8 times for $20 \mathrm{~min}$ over a period of 4 weeks. However, Marchand et al. [10] found in patients with low back pain that TENS significantly decreased intensity ratings in a cumulative manner over repeated treatment sessions. So for shorter periods of TENS treatment, less pain reduction may be expected than for longer treatment periods.
Willingness to continue TENS is more commonly used to evaluate treatment effect in daily practice and is assumed to reflect the result of a balance between effort and therapeutic result [11]. After 3 months of TENS treatment, Eriksson et al. [12] found $50 \%$ pain relief or more in $72 \%$ of the patients with chronic pain, who continued to use TENS after an initial short trial period. Therefore, willingness to continue TENS seems to be a preferable and clinically relevant outcome measure to evaluate shortterm effects of TENS.

The aim of this study is to test the efficacy of shortterm TENS treatment with respect to these outcome measures. Our primary research question is: is there a difference in the proportions of patients with chronic pain, satisfied with the initial treatment result and willing to continue TENS or sham TENS, after a short treatment period? Our secondary questions are: is there a difference in relief in pain intensity between patients treated with TENS or sham TENS, and do patients treated with TENS or sham TENS and who are satisfied with the initial treatment result differ in relief in pain intensity from each other and from those who do not continue treatment?

\section{Methods}

\section{Design}

We performed a prospective, randomised and controlled trial comparing TENS and sham TENS. A concealed block-wise randomisation procedure was used, and patients, therapists and research assistants were blinded for treatment allocation.

\section{Subjects}

Patients with chronic pain participating in this study were referred to the Pain Centre of the Radboud University Medical Centre Nijmegen by their family doctor (approximately $30 \%$ ) or by a medical specialist (approximately 70\%). Anaesthesiologists and physiotherapists of the Pain Centre screened patients for TENS treatment.

Patients were eligible for this study if they met the inclusion criteria. Inclusion criteria were: (1) patients with chronic pain of benign origin referred to the Pain Centre, (2) duration of pain $>6$ months, (3) age above 18 years. Exclusion criteria were: (1) previous TENS treatment (because this could affect sham TENS credibility [13]), (2) pain in face or head (because visible electrode placement might affect compliance, and hair could impair optimal electrode placement), (3) several different pain sites (because of the limited area TENS electrodes can serve), (4) history of a cerebral vascular accident (because possible spinothalamocortical pathway damage could affect the outcome of TENS - and possibly sham TENS treatment, too), (5) no 
relatives/friends to help replace electrodes, thus jeopardising optimal TENS use), (6) involvement in ongoing litigation because of their pain [11] and (7) psychological intervention proposed by the Pain Centre psychologists (this would interact with TENS treatment outcome in an unpredictable way, and withholding it would be unethical). Eligible patients were included in this study after signing informed consent. The Central Committee on Research Involving Human Subjects approved this study.

\section{Apparatus}

For TENS and sham TENS treatment, identical devices (ELPHA II 1000, Danmeter A/S, Denmark) were used, which were specially prepared for this study. On the devices' display, the current intensity level was visible with a warning indicator for low battery load. Stimulation pulse frequency was set to $80 \mathrm{~Hz}$ and pulse width to $50 \mu$ s for both channels (high-frequency TENS). Pulses were delivered with an asymmetrical biphasic waveform in a continuous pattern. Maximum output was $40 \mathrm{~mA}$ constant current at a minimum of $2.0 \mathrm{kOhm}$ electrode resistance. Sham TENS devices showed a maximum of 10 or $20 \mathrm{~mA}$ on the display, but no current was actually delivered to the electrodes. Duration of actual use of the device (referred to as "compliance") was registered and could be read out by pressing a special key combination. Patients were not aware of this feature. Disposable $5 \times 6.4 \mathrm{~cm}$ self-adhering electrodes were used with an active area of $6.5 \mathrm{~cm}^{2}$.

\section{General procedure}

Patients eligible for the TENS treatment received written information in which they were asked to participate in the study. In the letter, it was explained that TENS seems to be effective at high and low intensities, and that treatment would be by one of these two options. There would also be a chance of receiving a sham TENS device in which the settings of pulses were neither effective nor harmful.

After inclusion, baseline measures were carried out and details of the TENS treatment procedure were explained. Patients were told that "chronic pain is due to changes in the central nerve system which function as an amplifier for pain stimuli, and that TENS would serve as an inhibitory control button for this amplifier by using specific mild stimulation". For seven days before planned start of treatment, patients had to fill in a VAS for pain at the same time every day, starting on Friday.

The next Friday - when treatment was started - the patient visited the physiotherapist for electrode application and for instruction on both TENS treatment modalities. Electrodes were applied over the superficial cutaneous nerves in the painful segment(s), determined by the physiotherapist, who also showed how to replace them accurately if necessary [14]. Next the physiotherapist let the patient experience the tingling (not pricking) sensation accompanying "high intensity" TENS. Stimulation intensity was then decreased to determine the level at which the patient did not experience any sensation. This was explained to be "low intensity" TENS. This intensity was adjusted to 10 or $20 \mathrm{~mA}$ by the physiotherapist (but not visible for the patient) if necessary, and the setting shown to the patient to make the patient believe that at 10 or $20 \mathrm{~mA}$ he or she would not experience any sensation.

Once acquainted with the method of treatment, the patient left the room and visited the research assistant whose only task was to deliver the high frequency or sham TENS device to the patient, as determined by the randomisation list. With a written instruction how to use the device and not to change pain medication the patient left the outpatient clinic. Ten days later, the patient returned for evaluation of the treatment effect. Before visiting the researcher, the receptionist asked the patient to leave the "TENS" device with her, "to check it for proper function". The reason was twofold: the receptionist could register the TENS use, and the researcher would not have access to the TENS device.

\section{Outcome measures}

The primary outcome was the proportion of patients satisfied with the initial treatment result and willing to continue treatment (yes or no).

The secondary outcome measure was pain intensity. Pain intensity was measured using a 10-cm VAS, ranging from no pain at all to the most intense pain imaginable [15]. Patients were instructed to rate their pain from that particular moment at the same time every day for a period of 14 days, starting one week before treatment. Multiple measures of pain intensity across time maximise the reliability and validity of pain assessment [16].

\section{Compliance and placebo credibility}

Duration of TENS use served as a measure for compliance. Data collection for TENS use was performed by the receptionist while the patient visited the researcher for treatment evaluation.

During evaluation of short-term effects, patients were asked how certain they felt about having a TENS or sham device using a questionnaire according to Deyo et al. [13].

\section{Sample size}

Two main evaluations were planned for this study: a short-term evaluation after ten days of treatment and a long-term evaluation after one year of treatment. The number of patients in each treatment group was based on long-term outcome. Assuming that $35 \%$ of the patients in the TENS group and $15 \%$ in the sham TENS group would be successfully treated after one year, then 72 patients per group would be needed to show this difference for a power of $80 \%$ and a two-sided alpha value of $p=0.05$ (chi square test). Allowing for dropouts, this suggested the inclusion of about 80 patients per group. 
Statistical methods

All analyses were based on the intention-to-treat population, defined as all randomised patients that started with treatment. The level of significance used was 0.05 . The primary outcome parameter was the proportion of patients willing to continue (sham) TENS (yes or no). The difference between the two treatment groups was tested with the chi square test. The second outcome parameter was the difference in the time course of the VAS score during the first treatment week and the mean of the VAS score in the baseline week. These were analysed using a mixed repeated measures model, including as fixed factors time (day), treatment (TENS and sham TENS) and all their first-order interactions, and furthermore a random intercept per patient. In addition, we performed secondary, post hoc testing for the differences in VAS time courses between patients continuing and not continuing treatment in the two treatment groups using a mixed repeated measures model, including the fixed factors treatment (TENS and sham TENS) and result of treatment (continuing treatment or not), the repeated measures factor time (day) and all their first-order interactions, and furthermore a random intercept per patient. Differences in compliance were analysed using Wilcoxon's two-sample test. The relationship between patients' opinion about device type and real TENS or sham TENS use was analysed using a chi square test. For the strength of the associations, the phi coefficient was calculated. Phi is interpreted as a Pearson's $r$ and phi squared is the proportion of the total distribution of the variables measured, which is explained by the relationship.

\section{Results}

\section{Subjects}

From January 2000 until January 2003, 406 consecutive patients were proposed for TENS treatment. Two hundred and three patients were excluded. One hundred and sixtyfive patients signed informed consent. Two of the included patients withdrew before the actual treatment took place; they were both assigned to the TENS group. Therefore, 163 patients entered this study (for details see Fig. 1).

The main pain diagnoses were: nerve injury, compression or surgery and diabetic neuropathy $(n=17)$; dorsal root injury, compression or surgery $(n=24)$; articular degeneration and osteoporosis of the spine $(n=47)$; articular degeneration of hip, knee or ankle $(n=5)$; unclassified limb or back pain $(n=10)$; bursitis and tendonitis $(n=12)$; postfracture or postsurgery pain $(n=33)$; visceral pain $(n=7)$; and whiplash injury $(n=8)$.

Demographic data and pain characteristics were similar for the TENS and sham TENS groups (Tables 1 and 2). The baseline week's time course for pain VAS values did not differ between the TENS and sham TENS groups.
Baseline VAS time course was also similar for patients willing to continue treatment or not (see Fig. 2).

\section{Outcome}

The proportion of patients willing to continue treatment differed significantly $\left(x^{2}=3.8, p=0.05\right)$ for TENS $(58 \%)$ compared to sham TENS $(42.7 \%)$. Pain intensity was significantly reduced for factor time $(p<0.001)$, but not for treatment modality $(p=0.53)$ or interaction of time and treatment modality $(p=0.52)$ during the treatment week. With post hoc testing, pain intensity for patients willing to continue treatment differed significantly (in time and magnitude; $p<0.001)$ from patients not willing to continue treatment, only for the treatment week. Mean proportion of relief in pain intensity for patients willing to continue treatment was $28.5 \%$ (SEM=3.9\%), 27.0\% (SEM=5.0) for the TENS group and $30.6 \%(S E M=6.5)$ for the sham TENS group. Pain intensity increased by $1 \%(\mathrm{SEM}=8.0)$ in the TENS group and $10 \%(\mathrm{SEM}=5.0)$ in the sham TENS group, for patients not willing to continue treatment. There were, however, again no differences according to treatment modality. The time course of pain intensities is illustrated in Figure 2.

\section{Compliance and placebo credibility}

Compliance data were missing in two patients, both in the TENS group; from one because of a device handling mistake during retrieval of the data, and from another due to technical TENS device failure. There was no significant difference in compliance, measured via duration of device operation, between the TENS and sham TENS groups $(p=0.89)$. However, in patients not willing to continue treatment, the sham TENS group used the device for slightly longer than the TENS group ( $p=0.04$; see Table 3 ).

We found a significant relationship between patients' opinion about having a sham or real TENS device and actually having a sham or real TENS device $\left(x^{2}=13.2\right.$, $p<0.001$, phi=0.28).

\section{Discussion}

The proportion of patients satisfied with treatment results and willing to continue treatment was greater in the TENS group compared to the sham TENS group. However, we found no difference in pain intensity over time between patients receiving TENS or sham TENS. Pain intensity 


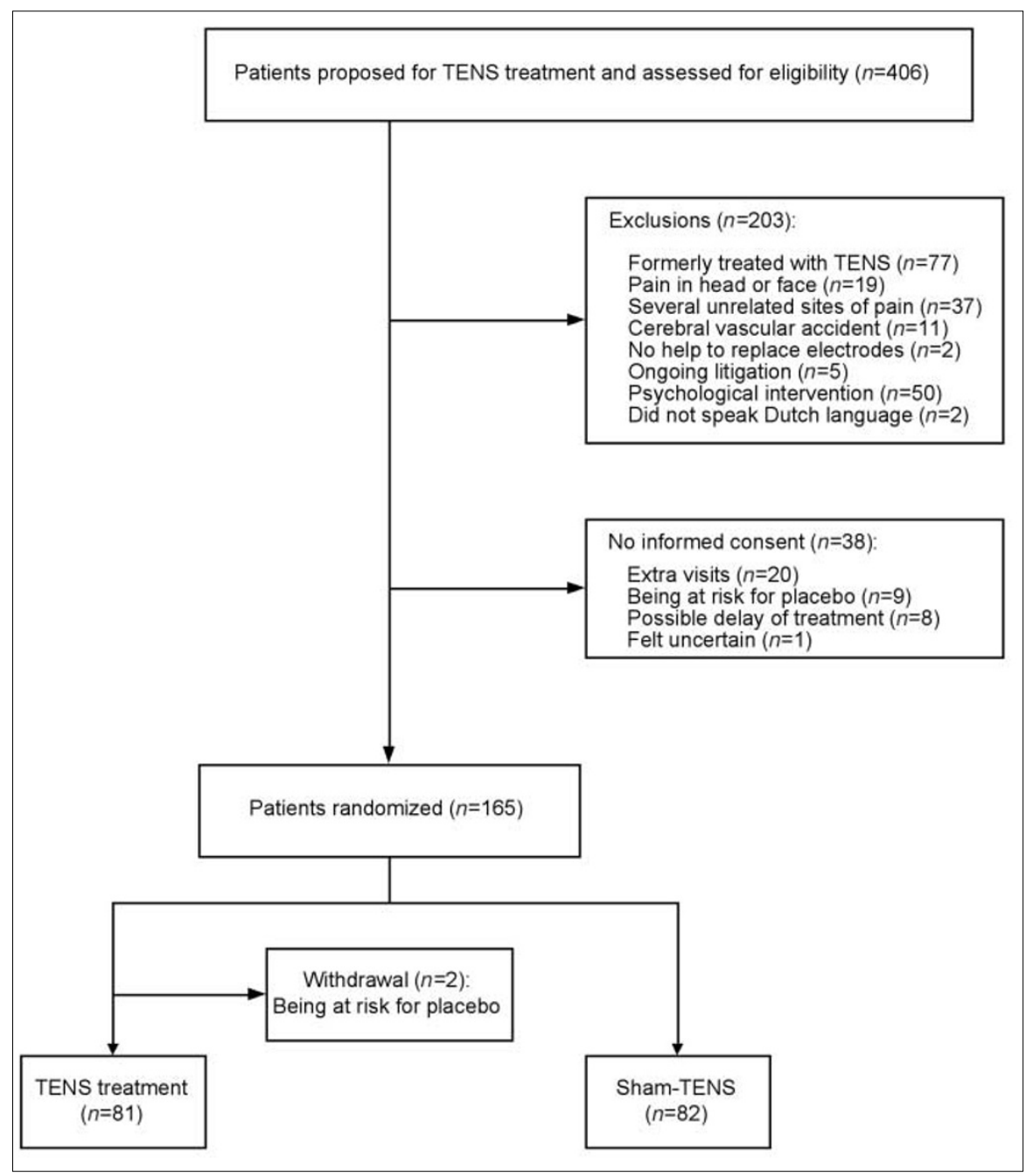

Fig. 1 Trial profile

Table 1 Baseline demographic details

TENS $(n=81)$

Sham TENS $(n=82)$

Total $(n=163)$

Age

Mean \pm SE (years)

$48.4 \pm 1.6$

$51.6 \pm 1.4$

$50.2 \pm 1.1$

Gender, $n(\%)$

Male

Female

33 (41)

33 (40)

$66(40)$

Nationality, $n(\%)$

Dutch

48 (69)

49 (60)

97 (60)

Others

79 (98)

$81(99)$

1 (1)

160 (98)

2 (2)

5 (6)

10 (12)

$71(87)$

Married/partner

67 (83)

1 (1)

4 (5)

3 (2)

Divorced

3 (4)

2 (3)

15 (9)

138 (85)

5 (3)

Widow(er)

13 (16)

54 (67)

15 (18)

5 (3)

Elementary school

Secondary education

12 (15)

Higher education

2 (2)

59 (72)

28 (17)

113 (69)

8 (10)

20 (13)

0 (0)

2 (1) 
Table 2 Baseline pain characteristics

\begin{tabular}{|c|c|c|c|}
\hline & TENS $(n=81)$ & Sham TENS $(n=82)$ & Total $(n=163)$ \\
\hline \multicolumn{4}{|l|}{ Intensity of pain (VAS, 0-100 mm) } \\
\hline Mean \pm SE & $62.2 \pm 2.1$ & $61.5 \pm 2.0$ & $61.9 \pm 1.4$ \\
\hline \multicolumn{4}{|l|}{ Duration of pain (years) } \\
\hline Mean \pm SE & $6.2 \pm 0.8$ & $6.6 \pm 0.9$ & $6.4 \pm 0.6$ \\
\hline Median (range) & $3.6(0.6-42.1)$ & $4.1(0.2-58.7)$ & $3.7(0.2-58.7)$ \\
\hline \multicolumn{4}{|l|}{ Variation of pain during the day, $n(\%)$} \\
\hline \multicolumn{4}{|l|}{ Almost constant pain } \\
\hline Constant, not changing & $29(36)$ & $18(22)$ & $47(29)$ \\
\hline Constant, increasing during the day & $30(37)$ & $39(48)$ & $69(42)$ \\
\hline Constant, decreasing during the day & $1(1)$ & $1(1)$ & $2(1)$ \\
\hline \multicolumn{4}{|l|}{ Variable pain } \\
\hline Variable pain, no pain free moments & $16(20)$ & $18(22)$ & $34(21)$ \\
\hline Variable pain, with pain free moments & $5(6)$ & $4(4)$ & $9(5)$ \\
\hline$\underline{\text { Other }}$ & $0(0)$ & $2(2)$ & $2(1)$ \\
\hline \multicolumn{4}{|l|}{ Origin of pain, $n(\%)$} \\
\hline Accident & $23(28)$ & $18(22)$ & $41(25)$ \\
\hline Surgery & $19(23)$ & $22(27)$ & $41(25)$ \\
\hline Disease & $6(7)$ & $11(13)$ & $17(10)$ \\
\hline Injury & $5(6)$ & $4(5)$ & $9(6)$ \\
\hline Long period of overstraining oneself & $9(11)$ & $14(17)$ & $23(14)$ \\
\hline Other & $2(2)$ & $0(0)$ & $2(1)$ \\
\hline Not known by patient & $17(21)$ & $13(16)$ & $30(18)$ \\
\hline \multicolumn{4}{|l|}{ Pain course, $n(\%)$} \\
\hline Gradually & $25(31)$ & $30(37)$ & $55(34)$ \\
\hline Sudden onset & $56(69)$ & $52(63)$ & $108(66)$ \\
\hline \multicolumn{4}{|l|}{ Recurrent pain, $n(\%)$} \\
\hline Yes, I had these complaints before & $38(47)$ & $38(47)$ & $76(47)$ \\
\hline No, this was the first time & $43(53)$ & $43(52)$ & $86(52)$ \\
\hline
\end{tabular}

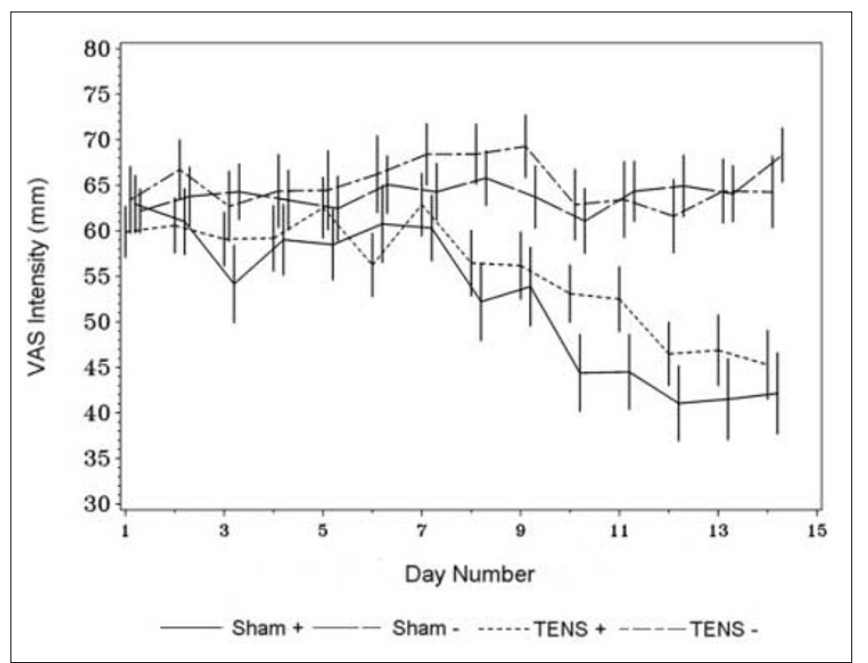

Fig. 2 Time course of VAS scores (mean \pm SEM) for satisfied patients $(+)$ and non-satisfied patients (-) of the TENS and sham TENS group, during baseline (day 1-7) and treatment periods (day 8-14)

equally decreased during both treatment applications, only for patients satisfied with treatment result and willing to continue treatment. For patients not willing to continue treat-
Table 3 Compliance; (sham) TENS use in hours per day

\begin{tabular}{llccc}
\hline Treatment & Satisfied? & $n$ & Missing & Mean (SD) \\
\hline Sham TENS & No & 47 & 0 & $11.6^{*}(4.9)$ \\
TENS & No & 33 & 1 & $9.8^{*}(2.7)$ \\
Sham TENS & Yes & 35 & 0 & $11.0(3.7)$ \\
TENS & Yes & 46 & 1 & $11.5(4.4)$ \\
\hline
\end{tabular}

$* p=0.04$ for difference in compliance between sham TENS and TENS group for patients not satisfied with treatment result

ment, pain intensities did not differ from baseline values. As shown in Figure 2, the results of real/sham TENS appeared to be initiated right from the start of treatment and to increase with time, during repeated treatment applications.

Comparison of our results with those of other TENS studies in chronic pain

In our study $58 \%$ of the patients were willing to continue TENS after an initial short trial period, which is in accord 
with the results of Köke et al. [17], who found $56 \%$ of the patients willing to continue TENS treatment after a trial period of two weeks.

For those patients willing to continue treatment, pain intensity decreased, on average, equally for TENS and sham TENS, by $28.5 \%$ compared to baseline, which is comparable to the results of Köke et al. [17], who found for patients with a positive assessment an average $33.5 \%$ pain reduction after two weeks of TENS treatment. Other reported relative differences in percentage change in pain severity from baseline ranged from $11 \%$ to $38 \%$ [18]. For the TENS treatment group as a whole, we found an average pain reduction of $16 \%$, which falls within this range.

Perhaps more important is the fact that our findings clearly show that TENS significantly decreased pain intensity ratings in a cumulative manner over repeated treatment sessions, in contrast to animal research, where repeated TENS administration results in gradual diminution of analgesic effect via opioid tolerance [19], but (except for the sham TENS) in line with the results of Marchand et al. [10]. However, how much the pain results will improve when extending the treatment period needs to be investigated. These findings may provide an explanation for the inconclusive results for TENS in chronic pain [1], as duration and frequency of TENS stimulation sessions vary largely between studies and, in general, stimulation periods were short and frequency of application was low [2]. Osiri et al. [20] found, as a result of reviewing TENS studies in osteoarthritis, that a significant difference in pain relief was achieved in studies with an intervention period of TENS application of at least 4 weeks.

In our study we applied high-frequency TENS with a frequency of $80 \mathrm{~Hz}$, a short pulse width of $50 \mu \mathrm{s}$ and lowintensity stimulation to selectively stimulate A-beta afferent neurons in the segment(s) of pain. This was assured by making the patient experience the different sensations of tingling (A-beta fibres) and pricking (nociceptive A-fibres) [21]. Patients used their device on average from 9.8 to 11.6 $\mathrm{h} /$ day, which is common in the treatment of chronic pain $[3,22]$. Whether different application parameters for TENS would have improved our results is questionable. Köke et al. [17] used high-frequency TENS comparable with our TENS application and found no difference in results of either high- or low-intensity stimulation. Nash et al. [23] compared continuous and pulsed high- and lowfrequency TENS at multiple intensity levels with a maximum just tolerated by the patients; they found however no differences in effect for the different TENS applications. In both studies, like in our study, patients were treated for various pain conditions. Recently, Defrin et al. [24] found noxious high-frequency stimulation to be more effective than innocuous stimulation in patients with osteoarthritis.
However, they used a non-portable device with a stimulation frequency of $4000 \mathrm{~Hz}$, which makes it less comparable with the standard TENS application.

\section{Differences regarding outcomes}

Our results suggest that pain intensity measured by VAS versus asking the patient's opinion about treatment result represent different, albeit related, pain outcome endpoints. As we found no differences in pain improvement by VAS scores between the TENS and sham TENS group, it would seem that patients' opinions about treatment results reflect the additional integration of factors apart from pain in assessing the clinical success of treatment. However it should be noted that VAS scores for pain intensity were measured once a day at a fixed time, reflecting the pain intensity at that particular moment, whereas patients' opinions about treatment result should include all improvements experienced by the patient during the whole treatment period. Interestingly, for patients satisfied with treatment results and willing to continue treatment, the mean pain reduction was $28.5 \%$, a clinically important difference [25], whereas for patients not willing to continue treatment there was no improvement in pain intensity during the treatment period as compared to the baseline period. Regarding the accuracy of pain integration into this assessment, it is interesting to note that Coghill et al. [26] found that individuals with similar activation patterns of the somatosensory, the anterior cingulate and prefrontal cortex provided similar subjective reports of pain magnitude. Finally, our findings seem to support the theory that patients either do or do not respond to TENS and sham TENS.

Specific versus non-specific effects of TENS treatment

Our results show that, for patients willing to continue treatment, there is no difference in relief in pain intensity between TENS and sham TENS, suggesting the same working mechanism, possibly a placebo response. Placebo effects can be very impressive; Evans found placebo to be as effective as a standard dose of morphine in 56\% of subjects [27]. Placebo response in TENS treatment has been said to range from less than $40 \%$ to more than $60 \%$ [28]. However, Vase et al. [29], studying chronic irritable bowel syndrome patients, found that the addition of a verbal suggestion for pain relief could increase the magnitude of placebo analgesia to that of an active agent. Therefore, it cannot be exclud- 
ed that the similar pain reductions achieved in the two groups are accomplished by different mechanisms. In this case it is interesting to know that in animal research high-frequency TENS decreased central sensitisation induced by inflammation in spinal dorsal horn neurons by activating delta-opioid receptors (for review see [30]), and in contrast, the placebo response in man is accomplished by the activation of mu-opioid receptors [7, 31]. However, further research in the underlying working mechanisms of TENS compared to sham TENS in man seems warranted.

\section{Placebo credibility}

The credibility of sham TENS has been challenged because patients do not feel the typical buzzing sensation of high-frequency TENS $[1,2]$. In the present study, patients' belief regarding real versus sham TENS was related to the actual use of real or sham TENS. However, we believe that the present results do provide support for the credibility of our version of sham TENS. Firstly, the strength of the relation was weak (phi $=0.28$; resulting in a shared variance of approximately $8 \%$ ). Secondly, there was no difference in actual use (compliance) between the real and sham TENS devices - and this device use was registered without the knowledge of the patient. Furthermore, subgroup analysis demonstrates that, on average, patients not willing to continue treatment as a whole used their TENS device approximately $1 \mathrm{~h} 45 \mathrm{~min}-$ utes less per day than sham TENS users. We would therefore suggest that our patients treated sham TENS as a credible treatment modality.

\section{Critique of study design}

The strong point of the present study is that we have succeeded in completing a large prospective, randomised, controlled and double-blind clinical trial of TENS treatment for chronic pain. In particular, a concerted effort was made to ensure that the sham TENS used was credible, and that the blinding of patients, therapists and researchers was maintained throughout the study. The fact that we did not include a natural history group might be considered a weak point, because pain improvement during treatment could have been attributed to the natural course of the pain. However, patients referred to the Pain Centre had to wait for three months before being seen, during which time their pain remained stable. Furthermore, pain reduction started promptly with the beginning of treatment (see Figure 2). It should be noted that adding a third natural history group to the study design would have reduced the probability of benefit for the volunteers. Thus patients would have entered the study with low expectations, violating the external validity of the study [32].

Our choice of the outcome measures may be considered a weak point, as they do not fully reflect all dimensions of chronic pain as proposed by the IMMPACT recommendations [33]. However, after this short treatment period we did not expect to achieve clinically relevant changes in disabilities. In this respect it is of importance to mention that Fishbain et al. [22] found that patients with chronic pain, who continued to use TENS after a trial period, improved by less pain interference with work, home and social activities, and increased activity level and pain management, after more than 6 months of TENS treatment. Whether these long-term results of TENS treatment differ from sham TENS still needs to be investigated.

Finally, the results of this study imply that those patients that experience relief by daily TENS (or sham TENS) application, expressed by willingness to continue treatment, improve by means of a significant pain reduction and that this amount of pain reduction is dependent on the period of stimulation. These results may have important implications for future TENS studies and clinical application, when defining response to treatment by means of a percentage change of pain intensity.

\section{Conclusions}

After ten days of TENS or sham TENS application, the proportions of patients satisfied with treatment results and willing to continue treatment differed significantly in favour of TENS. However, no differences in pain intensity were found for patients treated with TENS or sham TENS. Only for patients satisfied with treatment result, pain intensity gradually decreased equally both for TENS and sham TENS with repeated treatment application. In conclusion, we found that the efficacy of short-term TENS treatment depends on the choice of outcome measure and the period of stimulation.

Further research is needed to explore the mechanisms underlying the effects of TENS versus sham TENS in man.

Acknowledgement This study was funded by The Netherlands Organization for Health Research and Development Grant 94031-053. We further wish to thank Wim Lemmens from the Department of Epidemiology \& Biostatistics for handling the data. 


\section{References}

1. Carroll D, Moore RA, McQuay HJ, Fairman F, Tramer M, Leijon G (2004) Transcutaneous electrical nerve stimulation (TENS) for chronic pain (Cochrane Review). In: The Cochrane Library Issue 3. John Wiley \& Sons, Ltd., Chichester, UK

2. McQuay HJ, Moore RA (1998)

Transcutaneous electrical nerve stimulation (TENS) in chronic pain. In: McQuay HJ, Moore RA (eds) An evidence-based resource for pain relief. Oxford University Press, Oxford, pp 207-211

3. Johnson MI, Ashton CH, Thompson JW (1991) An in-depth study of longterm users of transcutaneous electrical nerve stimulation (TENS). Implications for clinical use of TENS. Pain 44:221-229

4. Moore SR, Shurman J (1997) Combined neuromuscular electrical stimulation and transcutaneous electrical nerve stimulation for treatment of chronic back pain: a double-blind, repeated measures comparison. Arch Phys Med Rehabil 78:55-60

5. Sluka KA, Bailey K, Bogush J, Olson R, Ricketts A (1998) Treatment with either high or low frequency TENS reduces the secondary hyperalgesia observed after injection of kaolin and carrageenan into the knee joint. Pain 77:97-102

6. Somers DL, Clemente FR (1998) High-frequency transcutaneous electrical nerve stimulation alters thermal but not mechanical allodynia following chronic constriction injury of the rat sciatic nerve. Arch Phys Med Rehabil 79:1370-1376

7. Benedetti F, Amanzio M (1997) The neurobiology of placebo analgesia: from endogenous opioids to cholecystokinin. Prog Neurobiol 52:109-125

8. Farrar JT, Portenoy RK, Berlin JA, Kinman JL, Strom BL (2000) Defining the clinically important difference in pain outcome measures. Pain 88:287-294

9. Smith CR, Lewith GT, Machin D (1983) TNS and osteo-arthritic pain. Preliminary study to establish a controlled method of assessing transcutaneous nerve stimulation as a treatment for the pain caused by osteo-arthritis of the knee. Physiotherapy 69:266-268
10. Marchand S, Charest J, Li J, Chenard JR, Lavignolle B, Laurencelle L (1993) Is TENS purely a placebo effect? A controlled study on chronic low back pain [see comments]. Pain 54:99-106

11. Lampl C, Kreczi T, Klingler D (1998) Transcutaneous electrical nerve stimulation in the treatment of chronic pain: predictive factors and evaluation of the method. Clin J Pain 14:134-142

12. Eriksson MB, Sjolund BH, Nielzen $S$ (1979) Long term results of peripheral conditioning stimulation as an analgesic measure in chronic pain. Pain 6:335-347

13. Deyo RA, Walsh NE, Schoenfeld LS, Ramamurthy S (1990) Can trials of physical treatments be blinded? The example of transcutaneous electrical nerve stimulation for chronic pain. Am J Phys Med Rehabil 69:6-10

14. Oosterhof J, Anderegg Q, Kersten H, Crul BJ (2003) TENS-protocol Pijncentrum UMC St Radboud. In: TENS bij chronische pijn. Handleiding voor fysiotherapeuten. Uitgeverij Coutinho, Bussum, pp 31-43

15. Revill SI, Robinson JO, Rosen M, Hogg MI (1976) The reliability of a linear analogue for evaluating pain. Anaesthesia 31:1191-1198

16. Jensen MP, McFarland CA (1993) Increasing the reliability and validity of pain intensity measurement in chronic pain patients [see comments]. Pain 55:195-203

17. Koke AJ, Schouten JS, LamerichsGeelen MJ et al (2004) Pain reducing effect of three types of transcutaneous electrical nerve stimulation in patients with chronic pain: a randomized crossover trial. Pain 108:36-42

18. Milne S, Welch V, Brosseau L et al (2004) Transcutaneous electrical nerve stimulation (TENS) for chronic low back pain (Cochrane review). In: The Cochrane Library Issue 3. John Wiley \& Sons, Ltd., Chichester, UK

19. Chandran P, Sluka KA (2003) Development of opioid tolerance with repeated transcutaneous electrical nerve stimulation administration. Pain 102:195-201
20. Osiri M, Welch V, Brosseau L et al (2004) Transcutaneous electrical nerve stimulation for knee osteoarthritis (Cochrane Review). In: The Cochrane Library Issue 3. John Wiley \& Sons, Ltd., Chichester, UK

21. Ziegler EA, Magerl W, Meyer RA, Treede RD (1999) Secondary hyperalgesia to punctate mechanical stimuli. Central sensitization to A-fibre nociceptor input. Brain 122:2245-2257

22. Fishbain DA, Chabal C, Abbott A, Heine LW, Cutler R (1996)

Transcutaneous electrical nerve stimulation (TENS) treatment outcome in long-term users. Clinical J Pain 12:201-214

23. Nash TP, Williams JD, Machin D (1990) TENS: does the type of stimulus really matter. Pain Clin 3:161-168

24. Defrin R, Ariel E, Peretz C (2005) Segmental noxious versus innocuous electrical stimulation for chronic pain relief and the effect of fading sensation during treatment. Pain 115:152-160

25. Farrar JT, Young JP Jr, LaMoreaux L, Werth JL, Poole RM (2001) Clinical importance of changes in chronic pain intensity measured on an 11-point numerical pain rating scale. Pain 94:149-158

26. Coghill RC, McHaffie JG, Yen YF (2003) Neural correlates of interindividual differences in the subjective experience of pain. Proc Natl Acad Sci USA 100:8538-8542

27. Evans G (1985) Expectancy, therapeutic instructions, and the placebo response. In: White L, Tursky B, Schwartz GE (eds) Placebo: Theory, Research, and Mechanisms. Guilford, New York, pp 215-228

28. Kaptchuk TJ, Goldman P, Stone DA, Stason WB (2000) Do medical devices have enhanced placebo effects? J Clin Epidemiol 53:786-792

29. Vase L, Robinson ME, Verne GN, Price DD (2003) The contributions of suggestion, desire, and expectation to placebo effects in irritable bowel syndrome patients. An empirical investigation. Pain 105:17-25 
30. Sluka KA, Walsh D (2003)

Transcutaneous electrical nerve stimulation: basic science mechanisms and clinical effectiveness. J Pain 4:109-121

31. Amanzio M, Benedetti F (1999)

Neuropharmacological dissection of placebo analgesia: expectation-activated opioid systems versus conditioningactivated specific subsystems. J

Neurosci 19:484-494
32. de-la-Fuente-Fernandez R, Schulzer M, Stoessl AJ (2002) The placebo effect in neurological disorders. Lancet Neurol 1:85-91
33. Dworkin RH, Turk DC, Farrar JT et al (2005) Core outcome measures for chronic pain clinical trials: IMMPACT recommendations. Pain 113:9-19 